Journal of Bioinformatics and Computational Biology

Vol. 16, No. 3 (2018) 1840012 (17 pages)

(C) The Author(s)

DOI: $10.1142 /$ S0219720018400127

\title{
Comparative analysis of probiotic bacteria based on a new definition of core genome
}

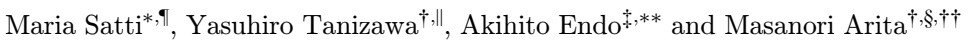 \\ *Department of Genetics, School of Life Science \\ SOKENDAI (The Graduate University for Advanced Studies) \\ Yata 1111, Mishima, Shizuoka 411-8540, Japan \\ ${ }^{\dagger}$ Center for Information Biology, National Institute of Genetics \\ Yata 1111, Mishima, Shizuoka 411-8540, Japan \\ \$Department of Food and Cosmetic Science, Faculty of Bioindustry \\ Tokyo University of Agriculture, 196 Yasaka \\ Abashiri, Hokkaido 099-2493, Japan \\ $\S_{\text {RIKEN Center for Sustainable Resource Science }}$ \\ 1-7-22 Suehiro-cho, Tsurumi-ku, Yokohama \\ Kanagawa 230-0045, Japan \\ "msatti@nig.ac.jp \\ lytanizaw@nig.ac.jp \\ **a3endou@bioindustry.nodai.ac.jp \\ †゙arita@nig.ac.jp
}

Received 30 November 2017

Revised 30 November 2017

Accepted 20 March 2018

Published 23 May 2018

\begin{abstract}
The commensal genus Bifidobacterium has probiotic properties. We prepared a public library of the gene functions of the genus Bifidobacterium for its online annotation. Orthologous gene cluster analysis showed that the pan genomes of Bifidobacterium and Lactobacillus exhibit striking similarities when mapped to the Clusters of Orthologous Group (COG) database of proteins. When the core genes in each genus were selected based on our statistical definition of "core genome", core genes were present in at least $92 \%$ of 52 Bifidobacterium and in $97 \%$ of 178 Lactobacillus genomes. Functional comparison of the core genes of the two genera revealed a significant difference in the categories "amino acid transport and metabolism" representing their difference in niche specificity. Over-represented Bifidobacterium protein families were primarily involved in host interactions, the complex compound metabolism, and in stress responses. These findings coincide with the published information and validate our bias-resilient definition of the core genome.
\end{abstract}

Keywords: Comparative genomics; metabolism; probiotics; pan-genome; core-genome.

TCorresponding author.

This is an Open Access article published by World Scientific Publishing Company. It is distributed under the terms of the Creative Commons Attribution 4.0 (CC-BY) License. Further distribution of this work is permitted, provided the original work is properly cited. 


\section{Introduction}

The Bifidobacterium was first isolated in 1899 by Henri Tissier from the feces of healthy breast-fed infants. ${ }^{1}$ It was initially named Bacillus bifidus ("divided into two parts" for its $Y$-shape), then Lactobacillus bifidus, and in 1924, Orla-Jensen categorized it into an independent genus Bifidobacterium. The genus now represents Gram-positive, immotile, non-gas-producing, obligatory anaerobic, and $Y$-shaped bacteria. ${ }^{1,2}$

Bifidobacterium species have been isolated from various ecological niches. In human, they are present in gastrointestinal tract (GIT) and oral cavity. They are the dominant clade of the gut microbiota of healthy breast-fed infants, ${ }^{3}$ and commensal species are considered important for microbial modulation at birth, e.g. the immune programming of its host. ${ }^{4,5}$ Most members of the genus are safe and nontoxic in humans. Bifidobacteria are generally host-specific and separated into human-, animal-, and insect-types. Human-type bifidobacteria are further classified as infant- (i.e. B. bifidum and B. breve) and adult-type (i.e. B. adolescentis and B. catenulatum). Certain species of bifidobacteria are found in environmental niches such as sewage, fermented products, and anaerobic digesters. ${ }^{6}$ Animal guts are suspected as the major source of these contaminants.

Earlier studies demonstrated that administration of certain species/strains of bifidobacteria elicits beneficial effects on host health; they include alleviation of atopic dermatitis and allergies, immune modulation, and shortening of diarrhea. ${ }^{7,8}$ Consequently, certain species/strains of the genus Bifidobacterium are used commercially as probiotics in the food industry, especially in dairy products. The genus is well adapted to the gut due to its ability to utilize host-derived oligosaccharides with sialic acid and human milk oligosaccharides (HMOs). ${ }^{9}$ Its characteristic metabolism is a pathway called the "bifidus shunt", which can produce 5 adenosine triphosphates (ATPs) per 2 glucose molecules (standard glycolysis produces 2 ATPs per 1 glucose) via fructose-6-phosphate phosphoketolase (EC 4.1.2.2). ${ }^{10}$ More than $8 \%$ of its total genes are involved in the carbohydrate metabolism. ${ }^{11}$ However, the specificity or particularity of these pathways remains to be investigated.

Large-scale sequencing can reveal the genomic characteristics of probiotic bacteria. The first complete genome in Bifidobacterium was reported for B. longum subsp.longum NCC2705. ${ }^{12}$ Subsequent comparative studies involved as many as 52 taxa comprised of 43 species and 9 subspecies. ${ }^{13-16}$ We now provide an accurate and consistent genomic annotation for this beneficial genus and compare its probiotic characteristics with the commensal probiotic genus, Lactobacillus. Our reference annotation library is freely available on our web annotation server. We also propose a new definition of the "core genome" and reveal genus-specific metabolic functions for Bifidobacterium and Lactobacillus.

From a bioinformatics perspective, our proposal for the "core genome" is an important step forward. Traditionally, its definition was "the set of genes included in all genomes" under investigation. Based on this definition, the size of the core genome decreases as the number of sequenced genomes increases. ${ }^{16}$ This data-dependent 
definition raises two problems: how to estimate the number of genomes required for determining the core genome, and how to identify the core genome among the obtained sequences. The first problem is solved easily. When the number of completely shared genes is plotted against the number of species, it drops sharply until the number of species is around 10; it then decreases very slowly as the number of species increases. This empirical knowledge implies that the core genome should be estimated from 10-20 genomes. It is more difficult to solve the second problem: how to identify the core genes in these genomes. If we posit that the core should be shared among all genomes, then genes for ribosomes and translation are over-represented. On the other hand, if we posit that conservation in $80 \%$ of the genomes delineates the core, spurious genes may slip in. Our simple criterion is to use the Clusters of Orthologous Group (COG) database to identify a stable core. This bias-resilient method is useful for many activities such as creating genome models, assessing type strains, and characterizing bacterial genus, especially when its member species contain very different genomes.

\section{Methods}

\subsection{Construction of the Bifidobacterium gene library}

Consistent genome annotation requires a reference library (pairs of sequences and their functional annotation) that is compatible with the published complete genome annotations. To prepare the library, we collected the complete genomes of $67 \mathrm{Bifi}$ dobacterium, 8 lactic acid bacteria strains and Bacillus subtilis contained in the NCBI Assembly Database (Table 1). The full genome sequences in the current study are available from GenBank. The protein sequences for all the genomes were extracted and subjected to orthologous clustering. Orthologous clusters were generated using GET_HOMOLOGUES software. ${ }^{17}$ The parameters for clustering were: $E$-value threshold of 10 e-5, minimum percentage coverage of $75 \%$, and Markov clustering algorithm using normalized BLAST scores (OrthoMCL). ${ }^{18} \mathrm{~A}$ total of 144,028 identified protein sequences were grouped into 21,255 clusters. The 12,545 singletons were discarded and the remaining 8710 clusters were analyzed. To obtain their protein names, gene symbols, and EC numbers, we referred to our existing library forLactobacillus. ${ }^{19}$ Among the 8710 clusters, we identified the functions of 6697 clusters. This indicates that close to $80 \%$ of the shared genes in Bifidobacterium have orthologs in Lactobacillus. The functions of the remaining 2013 gene clusters were sought manually by referencing the NCBI Conserved Domain Database (NCBICDD) using Reverse Position-Specific BLAST. This yielded that 15 Bifidobacterium genomes were close duplicates, and they were excluded from further analysis. The remaining 52 genomes were analyzed. Independently, the 45,038 gene clusters in 178 Lactobacillus genomes were obtained from our DAGA library. ${ }^{19}$ A newly sequenced Bifidobacterium can be easily annotated through this system at https://dfast.nig.ac.jp/. 
Table 1. The list of genomic sequences used for constructing the reference library.

\begin{tabular}{|c|c|c|c|c|}
\hline Accession & Bioproject & Biosample & TaxID & Organism \\
\hline A_000007525.1 & PRJNA328 & SAMN02603675 & 206672 & B. longum NCC2705 \\
\hline GCA_000008945.1 & PRJNA18773 & SAMN02603512 & 205913 & B. longum DJO10A \\
\hline GCA_000010425.1 & PRJNA16321 & SAMD00061080 & 367928 & B. adolescentis ATCC 15703 \\
\hline GCA_000020425.1 & PRJNA17189 & SAMN02598380 & 391904 & $\begin{array}{l}\text { B. longum subsp.infantis ATCC } \\
1569=\text { JCM } 1222=\text { DSM } 20088\end{array}$ \\
\hline GCA_000021425.1 & PRJNA19423 & SAMN02603485 & 442563 & B. animalis subsp. lactis AD011 \\
\hline GCA_000022705.1 & PRJNA32897 & SAMN02603172 & 580050 & B. animalis subsp. lactis $\mathrm{Bl}-04$ \\
\hline GCA_000022965.1 & PRJNA32893 & SAMN02603171 & 555970 & B. animalis subsp. lactis DSM 10140 \\
\hline GCA_000024445.1 & PRJNA17583 & SAMN02603177 & 401473 & B. dentium $\mathrm{Bd} 1$ \\
\hline GCA_000025245.1 & PRJNA42883 & SAMN02603131 & 552531 & B. animalis subsp. lactis BB-12 \\
\hline GCA_000092325.1 & PRJNA47579 & SAMN02603181 & 759350 & B. longum subsp. longum JDM301 \\
\hline GCA_000092765.1 & PRJNA32515 & SAMN02603934 & 573236 & B. animalis subsp. lactis V9 \\
\hline GCA_000164965.1 & PRJNA51963 & SAMN02603395 & 883062 & B. bifidum S17 \\
\hline GCA_000165905.1 & PRJNA42863 & SAMN02603510 & 702459 & B. bifidum PRL2010 \\
\hline GCA_000166315.1 & PRJNA53143 & SAMN02603469 & 890402 & B. longum subsp. longum BBMN68 \\
\hline GCA_000196555.1 & PRJDA32047 & SAMD00060951 & 565042 & B. longum subsp. longum JCM 1217 \\
\hline GCA_000196575.1 & PRJDA32051 & SAMD00060953 & 565040 & B. longum subsp. infantis $157 \mathrm{~F}$ \\
\hline GCA_000210755.1 & PRJNA45963 & SAMEA3138379 & 722911 & B. longum subsp. longum $\mathrm{F} 8$ \\
\hline GCA_000213865.1 & PRJNA51077 & SAMN00100758 & 866777 & B. breve ACS \\
\hline GCA_000219455.1 & PRJNA66401 & SAMN02603656 & 1035817 & B. longum subsp. longum KACC 91563 \\
\hline GCA_000220135.1 & PRJNA13487 & SAMN02604112 & 326426 & B. breve UCC2003 \\
\hline GCA_000220885.1 & PRJNA67865 & SAMN02604350 & 1042403 & B. animalis subsp. lac \\
\hline GCA_000224965.2 & PRJNA71815 & SAMN02604239 & 1075106 & B. animalis subsp. lactis $\mathrm{BLC} 1$ \\
\hline GCA_000265095.1 & PRJNA21077 & SAMN02603486 & 484020 & B. bifidum BGN4 \\
\hline GCA_000269965.1 & PRJDA32049 & SAMD00060952 & 391904 & $\begin{array}{l}\text { B. longum subsp. infantis ATCC } \\
\quad 15697=\text { JCM } 1222=\text { DSM } 20088\end{array}$ \\
\hline ט0007706 & $\mathrm{PI}$ & 14 & 290 & B. animalis subsp. lactis $\mathrm{B} 420$ \\
\hline GCA_000277345.1 & PRJNA156975 & SAMN02603215 & 742729 & B. animalis subsp. lactis $\mathrm{Bi}-07$ \\
\hline GCA_000304215.1 & PRJNA83029 & SAMN02604114 & 1147128 & B. asteroides PRL2011 \\
\hline GCA_000347695.1 & PRJNA179141 & SAMN02603226 & 1254439 & B. thermophilum RBL67 \\
\hline GCA_000414215.1 & PRJNA186412 & SAMN02604238 & 1281781 & B. animalis subsp. lactis $\mathrm{Bl} 12$ \\
\hline GCA_000568955.1 & PRJNA214949 & SAMN03081478 & 1385938 & B. breve $12 \mathrm{~L}$ \\
\hline GCA_000568975.1 & PRJNA214950 & SAMN03081479 & 1385939 & B. breve JCM \\
\hline GCA_000569015.1 & PRJNA214951 & SAMN03081480 & 1385940 & B. breve JCM \\
\hline GCA_000569035.1 & PRJNA214954 & SAMN03081481 & 1385941 & B. breve NCFB 2258 \\
\hline GCA_000569055.1 & PRJNA214955 & SAMN03081482 & 1385942 & B. breve $689 \mathrm{~b}$ \\
\hline GCA_000569075.1 & PRJNA214956 & SAMN03081483 & 936351 & B. breve $\mathrm{S} 27$ \\
\hline GCA_000695895.1 & PRJNA245929 & SAMN02797738 & 28025 & B. animalis \\
\hline GCA_000706765.1 & PRJNA206546 & SAMN05771123 & 1341694 & B. indicum LMG $11587=$ DSM 20214 \\
\hline GCA_000730205.1 & PRJNA251950 & SAMN02843216 & 216816 & B. longum \\
\hline GCA_000737865.1 & PRJNA239194 & SAMN02666223 & 1687 & B. coryneforme \\
\hline GCA_000737885.1 & PRJNA240293 & SAMN02673695 & 1680 & B. adolescentis \\
\hline GCA_000772485.1 & PRJNA191592 & SAMN03093230 & 1300227 & B. longum subsp. longum GT15 \\
\hline GCA_000800455.1 & PRJNA234365 & SAMN03253090 & 1447716 & B. kashiwanohense PV20-2 \\
\hline GCA_000800475.2 & PRJNA234364 & SAMN03253091 & 1447715 & B. pseudolongum PV8-2 \\
\hline GCA_000816205.1 & PRJNA225662 & SAMN02726251 & 1316911 & B. animalis subsp. lactis KLDS2.0603 \\
\hline GCA_000817045.1 & PRJNA271239 & SAMN03273367 & 28025 & B. animalis \\
\hline GCA_000817995.1 & PRJNA271241 & SAMN03273368 & 1680 & B. adolescentis \\
\hline GCA_000818055.1 & PRJNA252546 & SAMN02941905 & 302911 & B. animalis subsp. lactis \\
\hline GCA_000829295.1 & PRJDB3123 & SAMD00019943 & 216816 & B. longum \\
\hline GCA_000966445.2 & PRJNA278963 & SAMN03435124 & 1683 & B. angulatum \\
\hline GCA_001025135.1 & PRJDB56 & SAMD00061040 & 500634 & $\begin{array}{l}\text { B. bifidum ATCC } 29521= \\
\text { JCM } 1255=\text { DSM } 20456\end{array}$ \\
\hline GCA_001025155.1 & PRJDB55 & SAMD00061039 & 518635 & B. angulatum DSM $20098=\mathrm{JCM} 7096$ \\
\hline GCA_001025175.1 & PRJDB57 & SAMD00061041 & 518634 & B. breve DSM $20213=$ JCM 1192 \\
\hline
\end{tabular}


Table 1. (Continued)

\begin{tabular}{|c|c|c|c|c|}
\hline Accession & Bioproject & Biosample & TaxID & Organism \\
\hline GCA_001025195.1 & PRJDB58 & SAMD00061042 & 566552 & $\begin{array}{l}\text { B. catenulatum DSM } 16992= \\
\text { JCM } 1194=\text { LMG } 11043\end{array}$ \\
\hline GCA_001025215.1 & PRJDB61 & SAMD00061045 & 547043 & $\begin{array}{l}\text { B. pseudocatenulatum DSM } \\
\quad 20438=\text { JCM } 1200=\text { LMG } 10505\end{array}$ \\
\hline GCA_001042595.1 & PRJDB59 & SAMD00061043 & 1150423 & B. dentium JCM $1195=$ DSM 20436 \\
\hline GCA_001042615.1 & PRJDB60 & SAMD00061044 & 1150460 & $\begin{array}{l}\text { B. kashiwanohense JCM } 15439 \\
\text { = DSM } 21854\end{array}$ \\
\hline GCA_001042635.1 & PRJDB62 & SAMD00061046 & 1150461 & B. scardovii JCM $12489=$ DSM 13734 \\
\hline GCA_001263395.1 & PRJNA286033 & SAMN02442035 & 1437605 & B. actinocoloniiforme DSM 22766 \\
\hline GCA_001281305.1 & PRJNA270905 & SAMN03271683 & 1682 & B. longum subsp. infantis \\
\hline GCA_001281345.1 & PRJNA270902 & SAMN03271680 & 1681 & B. bifidum \\
\hline GCA_001293145.1 & PRJNA270904 & SAMN03271682 & 216816 & B. longum \\
\hline GCA_001446255.1 & PRJNA287223 & SAMN03785818 & 1679 & B. longum subsp. longum \\
\hline GCA_001446275.1 & PRJNA287222 & SAMN03785819 & 1679 & B. longum subsp. longum \\
\hline GCA_001688645.1 & PRJNA317592 & SAMN04621611 & 302912 & B. animalis subsp. animali $\mathrm{s}$ \\
\hline GCA_001719085.1 & PRJNA301703 & SAMN04254466 & 216816 & B. longum \\
\hline GCA_001725985.1 & PRJNA316119 & SAMN04576213 & 1679 & B. longum subsp. longum \\
\hline GCA_000006865.1 & PRJNA72 & SAMN02603339 & 272623 & Lactococcus lactis subsp. lactis Il 1403 \\
\hline GCA_000007785.1 & PRJNA70 & SAMN02603978 & 226185 & Enterococcus faecalis V583 \\
\hline GCA_000008925.1 & PRJNA13280 & SAMN02604111 & 362948 & Lactobacillus salivarius UCC118 \\
\hline GCA_000011985.1 & PRJNA82 & SAMN02603047 & 272621 & Lactobacillus acidophilus NCFM \\
\hline GCA_000203855.3 & PRJNA356 & SAMEA3138345 & 220668 & Lactobacillus plantarum WCFS1 \\
\hline GCA_000174395.2 & PRJNA30627 & SAMN00002237 & 333849 & Enterococcus faecium DO \\
\hline GCA_000006785.2 & PRJNA269 & SAMN02604089 & 160490 & Streptococcus pyogenes M1 GAS \\
\hline GCA_000014525.1 & PRJNA402 & SAMN02598528 & 321967 & Lactobacillus paracasei ATCC 334 \\
\hline GCA_000009045.1 & PRJNA76 & SAMEA3138188 & 224308 & Bacillus subtilis subsp. subtilis str. 168 \\
\hline
\end{tabular}

\subsection{Pan- and core-genome computation and COG assignment}

For pan- and core-genome analysis, we used COG functional categories. Gene clusters in the 52 Bifidobacterium and 178 Lactobacillus genomes were queried against NCBI-CDD using Reverse Position-Specific BLAST; the COG category was assigned with Perl script "cdd2cog" available at https://github.com/aleimba/bac-genomicsscripts/tree/master/cdd2cog. The entire cluster set represents the pan genome.

A commonly used definition of the "core" genome includes only genes that are present in all genomes. By this definition, the number of core genes constantly decreases as the number of genomes grow. Our concept of " $n$-core" entails the set of genes that is conserved in $n$ percent of the complete genomes. For example, in Bifidobacterium, the designation "100-core", "98-core", "96-core" , .., "83-core" indicates the set of genes conserved in all 52-, 51-, 50-, .., and 43 of the 52 genomes. We created 10 such $n$-cores (from 100- to 83-core). Each $n$-core exhibits a different distribution of 26 COG functional categories (the COG ratio) because the number of shared genes increased as $n$ decreased (Table 2). For example, "translation and ribosomal structure and biogenesis" category $(\mathrm{J})$ is the 5th largest group in the 96core, but it became the second in the 98-core, and the largest in the 100-core because as many as 27 and 76 genes in $\mathrm{J}$ are shared among 50 and 51 genomes, respectively (Table 2). Given a set of genomes, we want to choose an appropriate $n$-core that 
Table 2. Rank (rows) and number of different COG functional categories (cells) in the $10 n$-cores (columns) of Bifidobacterium (Color coding: dark: 10 or more gene additions; gray: $10>$ and $>=5$ gene additions; light gray: $5>$ and $>=2$ gene additions. Some gene numbers are presented as fractions because the same gene may fall in multiple COG categories).

\begin{tabular}{|c|c|c|c|c|c|c|c|c|c|c|c|c|c|c|c|c|}
\hline $\begin{array}{c}100 \text {-core } \\
(52)\end{array}$ & $\begin{array}{c}98 \text {-core } \\
(51)\end{array}$ & $\begin{array}{c}\text { 96-core } \\
(50)\end{array}$ & \multicolumn{2}{|c|}{$\begin{array}{c}\text { 94-core } \\
(49)\end{array}$} & \multicolumn{2}{|c|}{$\begin{array}{c}92 \text {-core } \\
(48)\end{array}$} & \multicolumn{2}{|c|}{$\begin{array}{c}90 \text {-core } \\
(47)\end{array}$} & \multicolumn{2}{|c|}{$\begin{array}{c}88 \text {-core } \\
(46)\end{array}$} & \multicolumn{2}{|c|}{$\begin{array}{c}87 \text {-core } \\
(45)\end{array}$} & \multicolumn{2}{|c|}{$\begin{array}{c}85 \text {-core } \\
(44)\end{array}$} & \multicolumn{2}{|c|}{$\begin{array}{c}\text { 83-core } \\
(43)\end{array}$} \\
\hline J 76 & E 29.5 & -16 & $\mathrm{E}$ & 16.8 & - & 8 & $\bar{K}$ & 5.5 & $\bar{G}$ & 9.3 & $\mathrm{G}$ & 5 & $\bar{G}$ & 9.3 & $\bar{G}$ & 3 \\
\hline L $\quad 25.5$ & J 27 & E 14.5 & & 6 & $\mathrm{~J}$ & 5.5 & G & 3 & I & 4 & S & 5 & $\mathrm{R}$ & 2 & V & 2 \\
\hline R 24.6 & R 19.5 & R 10.5 & G & 6 & $\mathrm{P}$ & 5.5 & S & 3 & G & 3 & $\mathrm{E}$ & 4.3 & S & 2 & K & 2 \\
\hline O 21.5 & -15 & L 9.5 & $\mathrm{C}$ & 5 & $\mathrm{R}$ & 5 & $\mathrm{R}$ & 2.5 & $P$ & 3 & $\mathrm{~F}$ & 3 & - & 2 & M & 1 \\
\hline G 21.3 & S 13 & J 8 & $\mathrm{R}$ & 4.5 & $\mathrm{~L}$ & 4 & V & 2 & $\mathrm{R}$ & 3 & $\mathrm{R}$ & 3 & D & 1 & $\mathrm{O}$ & 1 \\
\hline-21 & L 12.8 & F 7.5 & I & 4.3 & - & 4 & E & 2 & - & 3 & $\mathrm{H}$ & 2.5 & M & 1 & E & 1 \\
\hline M 20.5 & G 11 & P 6 & $\mathrm{~F}$ & 4 & $\mathrm{~T}$ & 3 & $\mathrm{~F}$ & 2 & M & 2 & $\mathrm{~K}$ & 2 & $\mathbf{J}$ & 1 & $\mathrm{~F}$ & 1 \\
\hline K 19 & C 9 & K 5 & $\mathrm{P}$ & 3.5 & K & 3 & $\mathrm{O}$ & 1.5 & $\mathrm{~J}$ & 1.5 & I & 2 & $\mathrm{C}$ & 1 & $\mathrm{P}$ & 1 \\
\hline E 19 & M 7.5 & S 5 & $\mathbf{J}$ & 3 & $S$ & 3 & $\mathrm{~L}$ & 1.5 & $\mathrm{E}$ & 1.5 & - & 2 & $\mathrm{~F}$ & 1 & $\mathrm{R}$ & 1 \\
\hline F $\quad 17.3$ & F $\quad 7.5$ & H 4.5 & S & 3 & $\mathrm{~F}$ & 2.5 & $\mathrm{H}$ & 1.5 & $\mathrm{~F}$ & 1.5 & $\mathrm{~L}$ & 1 & & 0.5 & S & 1 \\
\hline C 16.8 & O 7 & G 3 & - & 3 & M & 2 & $\mathrm{P}$ & 1.5 & D & 1 & $\mathrm{P}$ & 1 & $\mathrm{H}$ & 0.5 & D & 0 \\
\hline T 12 & I 6.3 & T 2.5 & $\mathrm{~T}$ & 2.5 & $\mathrm{O}$ & 2 & D & 1 & $\mathrm{~T}$ & 1 & M & 0.5 & $\mathrm{~N}$ & 0 & $\mathrm{~N}$ & 0 \\
\hline H 10 & T 6 & C $\quad 2.2$ & D & 2 & $\mathrm{C}$ & 2 & M & 1 & $\mathrm{~V}$ & 1 & $\mathrm{~N}$ & 0.5 & $\mathrm{O}$ & 0 & $\mathrm{~T}$ & 0 \\
\hline P 9 & K 5.5 & M 2 & $\mathrm{O}$ & 2 & G & 2 & $\mathrm{~T}$ & 1 & $\mathrm{C}$ & 1 & $\mathrm{U}$ & 0.5 & $\mathrm{~T}$ & 0 & $\mathrm{U}$ & 0 \\
\hline S 9 & U 5 & I 2 & $\mathrm{H}$ & 1.8 & $\mathrm{D}$ & 1 & $\mathrm{~J}$ & 1 & A & 0.5 & $\mathrm{~J}$ & 0.3 & $\mathrm{U}$ & 0 & W & 0 \\
\hline I 7 & P 4.5 & D 1.5 & $\mathrm{~K}$ & 1.5 & $\mathrm{H}$ & 1 & I & 1 & $\mathrm{~L}$ & 0.5 & $\mathrm{D}$ & 0 & $\mathrm{~V}$ & 0 & $\mathrm{Y}$ & 0 \\
\hline D 6 & D 4 & O 1 & $\mathrm{U}$ & 1 & $\mathrm{~N}$ & 0 & $\mathrm{~N}$ & 0.5 & $\mathrm{~N}$ & 0 & $\mathrm{O}$ & 0 & W & 0 & $\mathrm{Z}$ & 0 \\
\hline U 4.5 & H 3.3 & V 1 & A & 0.5 & $\mathrm{U}$ & 0 & A & 0.5 & $\mathrm{O}$ & 0 & $\mathrm{~T}$ & 0 & $\mathrm{Y}$ & 0 & A & 0 \\
\hline A 3.8 & V 2 & $\mathrm{~N} \quad 0.5$ & $\mathrm{~L}$ & 0.5 & $\mathrm{~V}$ & 0 & K & 0.5 & $\mathrm{U}$ & 0 & $\mathrm{~V}$ & 0 & $\mathrm{Z}$ & 0 & B & 0 \\
\hline V 3 & В 0.5 & U $\quad 0.5$ & $\mathrm{~N}$ & 0 & W & 0 & $\mathrm{U}$ & 0 & W & 0 & & 0 & A & 0 & $\mathbf{J}$ & 0 \\
\hline B 2.3 & $\mathrm{~N} \quad 0$ & A 0.5 & V & 0 & $\mathrm{Y}$ & 0 & W & 0 & $\mathrm{Y}$ & 0 & $\mathrm{Y}$ & 0 & B & 0 & $\mathrm{~L}$ & 0 \\
\hline Q 1 & W 0 & W 0 & W & 0 & $\mathrm{Z}$ & 0 & $\mathrm{Y}$ & 0 & $\mathrm{Z}$ & 0 & Z & 0 & K & 0 & $\mathrm{C}$ & 0 \\
\hline N 0.5 & Y 0 & Y 0 & $\mathrm{Y}$ & 0 & A & 0 & $\mathrm{Z}$ & 0 & B & 0 & A & 0 & $\mathrm{~L}$ & 0 & $\mathrm{H}$ & 0 \\
\hline Y 0.5 & $\mathrm{Z} 0$ & $\mathrm{Z} \quad 0$ & $\mathrm{Z}$ & 0 & B & 0 & B & 0 & $\mathrm{H}$ & 0 & B & 0 & I & 0 & I & 0 \\
\hline W 0 & A 0 & B 0 & B & 0 & I & 0 & $\mathrm{C}$ & 0 & Q & 0 & $\mathrm{C}$ & 0 & $\mathrm{P}$ & 0 & Q & 0 \\
\hline $\mathrm{Z} 0$ & Q 0 & Q 0 & Q & 0 & $\mathrm{Q}$ & 0 & Q & 0 & $\mathrm{~S}$ & 0 & $\mathrm{Q}$ & 0 & Q & 0 & - & 0 \\
\hline
\end{tabular}

avoids inappropriate deletions or inclusions. To choose the best $n$ value, we first created "consensus COG ordering" based on the majority rule whereby all COG categories were ordered according to their abundance in each $n$-core and assigned their ranks. Then, the COG categories were reordered by their average rank in 10 $n$-cores. We designated this COG ordering, computed from the averages, "consensus COG ordering". Next, each $n$-core was compared against the consensus COG ordering using the Spearman's rank correlation coefficient, and the closest core was chosen. In Bifidobacterium, 773 genes present in at least 48 of the 52 genomes (92-core) were selected as the consensus core. In Lactobacillus, 472 genes present in at least 172 of the 178 genomes (97-core) were identified as the consensus core.

\subsection{Odds ratio $(O R)$ and p-value of genus-specific functions}

Statistical analysis was performed to evaluate the relative abundance of protein families in Bifidobacterium and Lactobacillus species. To identify over- and underrepresented functional categories in both species, we calculated the OR using a twoby-two contingency table. It included four parameters based on the number of protein families that were (a) present or (b) absent in the respective COG category of Bifidobacterium, and (c) present or (d) absent in the respective COG category of Lactobacillus. The formula for calculating the OR was ad/bc. This value is also regarded as an approximated risk value, since the number of genes in each COG 
category is much smaller than the total. From the OR, we calculated the $95 \%$ confidence interval (CI) as follows:

$$
\begin{array}{ll}
\exp [\ln (\mathrm{OR})+1.96 \sqrt{ }(1 / a+1 / b+1 / c+1 / d)] & \text { for the upper limit, and } \\
\exp [\ln (\mathrm{OR})-1.96 \sqrt{ }(1 / a+1 / b+1 / c+1 / d)] & \text { for the lower limit. }
\end{array}
$$

When $\mathrm{D}$ is the difference in the natural $\log (\mathrm{ln})$ of the upper and the lower limits, the standard error (SE) is computed as $\mathrm{D} /(2 * 1.96)$, the $z$ score was $\ln (\mathrm{OR}) / \mathrm{SE}$. The corresponding $p$-value was obtained with $R$ function $2 * \operatorname{pnorm}(-\operatorname{abs}(z \operatorname{score}))$.

\subsection{Assignment of carbohydrate-active enzymes}

Carbohydrate-active enzymes were identified based on their similarity on the Carbohydrate Active Enzymes (CAZy) database. The online tools CAZymes Analysis Toolkit (CAT) and dbCAN were applied manually. ${ }^{20,21}$

\section{Results and Discussion}

\subsection{General genomic features of the genera Bifidobacterium and Lactobacillus}

The genome sequences of 52 (sub) species of Bifidobacterium and 178 (sub) species of Lactobacillus were retrieved from the NCBI Assembly database. The genome size of Bifidobacterium varied from $1.6 \mathrm{Mb}$ for $B$. commune to $3.3 \mathrm{Mb}$ for $B$. biavatii (Fig. 1(a)). The approximate G + C content ranged from $53-66 \%$ (Fig. 1(b)) and the approximate number of coding sequences (CDS) from 1200-2500 (Fig. 1(c)). The genome size of Lactobacillus ranged from $1.2 \mathrm{Mb}$ for L. sanfranciscensis to $3.7 \mathrm{Mb}$ for L. pentosus (Fig. 2(a)). The $\mathrm{G}+\mathrm{C}$ content of Lactobacillus was lower than that of Bifidobacterium, it ranged from 32-57\% (Fig. 2(b)).

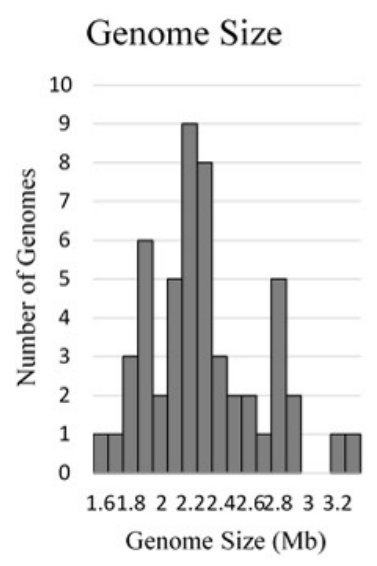

(a)

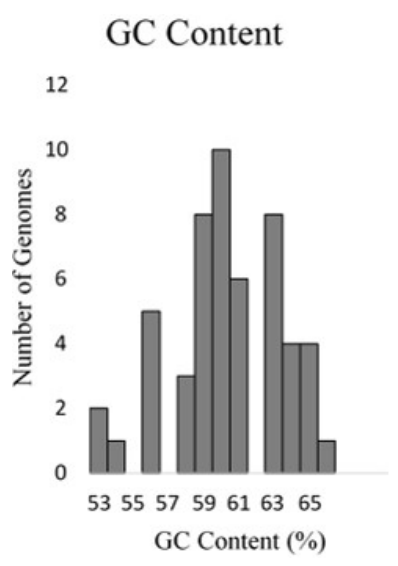

(b)

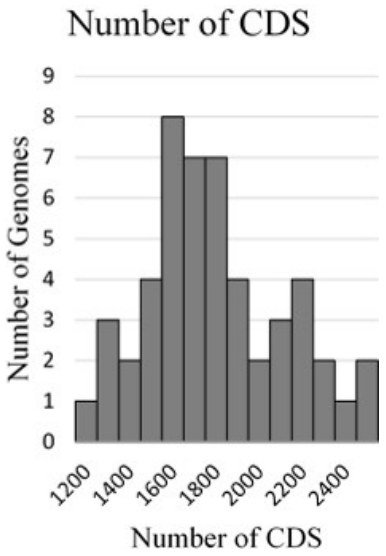

(c)

Fig. 1. Genomic features of genus Bifidobacterium. 


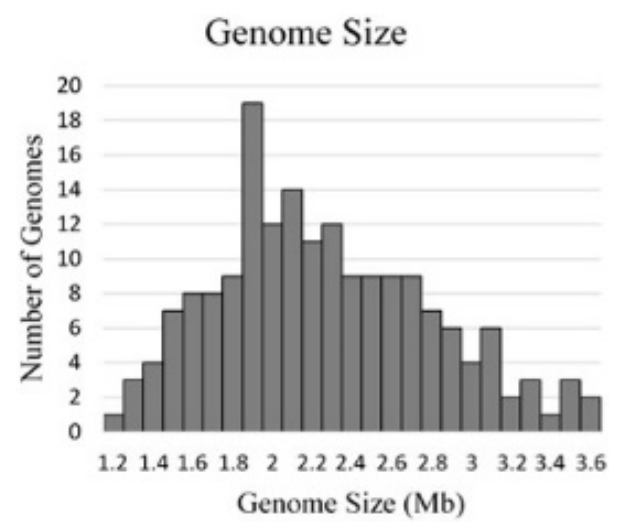

(a)

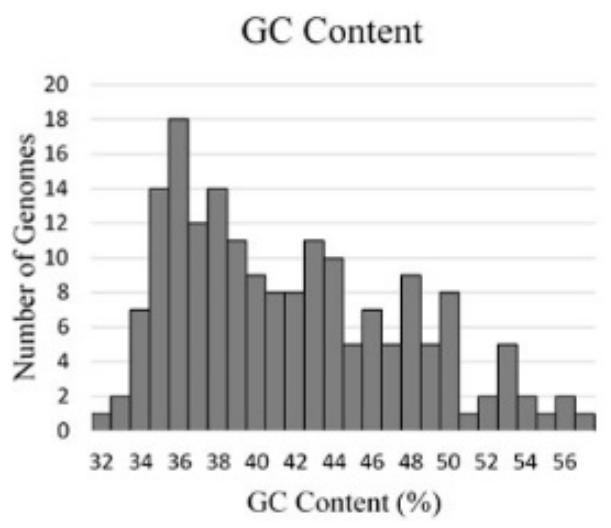

(b)

Fig. 2. Genomic features of genus Lactobacillus.

Phylogenetically, the two genera are distant. Lactobacillus, a low $\mathrm{G}+\mathrm{C}$ bacillus, belongs to the phylum Firmicutes, whereas Bifidobacterium belongs to the phylum Actinobacteria. However, they share a common niche habitat (animal gut), energy metabolism (lactate fermentation), and both are used industrially as probiotic species. Genome-scale comparison will reveal their metabolic similarities.

\subsection{COG comparison of the pan-and core-genomes of Bifidobacterium and Lactobacillus}

The pan- and core-genomes of the 52 Bifidobacterium and the 178 Lactobacillus were determined as described in Sec. 2. The pan genome of Bifidobacterium and Lactobacillus included 16,232 and 45,038 gene clusters, respectively. Functional categories for all gene clusters of both genera were assigned according to the COG classification. Almost $30 \%$ of the pan genomes (9283 and 26,944 gene clusters in Bifidobacterium and Lactobacillus, respectively) remained functionally unknown and assigned to no COG category (Fig. 3). This is common to many other bacteria; close to $30 \%$ of the genes of Escherichia coli are functionally unknown.

The overall COG classification of the pan genomes of the two distant genera was strikingly similar. In both, the highest fractions were identical as was the ratio of categories. Slightly different in Bifidobacterium and Lactobacillus were the "amino acid transport and metabolism" (E: $7 \%$ versus $5 \%$ ) and "carbohydrate transport and metabolism" (G: $8 \%$ versus $6 \%$ ).

The COG ratios of the core genomes were quite different. Figure 4 is a pie chart of the 92-core for Bifidobacterium and the 97-core for Lactobacillus. In the core genome of Bifidobacterium, more metabolism-related genes, especially (E), and in Lactobacillus more information storage and processing genes, especially "translation, ribosomal structure, and biogenesis" (J), are enriched. In the Bifidobacterium core 


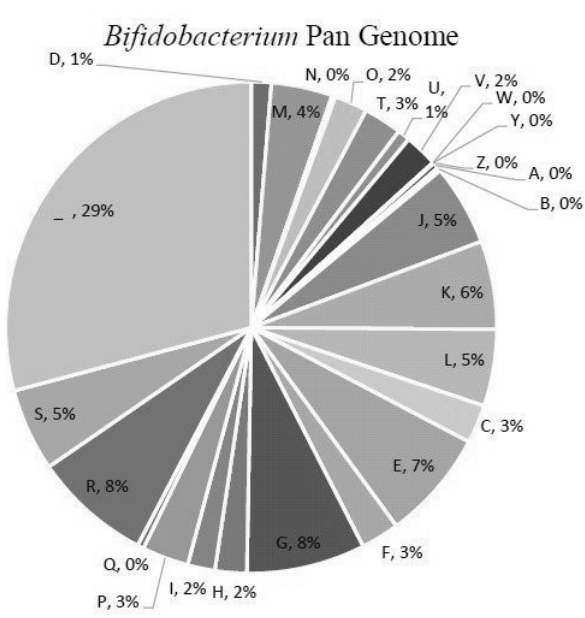

- [D] Cell cycle control, cell division, chromosome partitioning - [N] Cell motility

- [T] Signal transduction mechanisms

- [V] Defense mechanisms

- [Y] Nuclear structure

- [A] RNA processing and modification

- [J] Translation, ribosomal structure and biogenesis

" [L] Replication, recombination and repair

- [E] Amino acid transport and metabolism

- [G] Carbohydrate transport and metabolism

- [I] Lipid transport and metabolism

- [Q] Secondary-metabolite biosynthesis, transport, and catabolism

- [S] Function unknown

$[\mathrm{D}],[\mathrm{M}],[\mathrm{N}],[\mathrm{O}],[\mathrm{T}],[\mathrm{U}],[\mathrm{V}],[\mathrm{W}],[\mathrm{Y}],[\mathrm{Z}]$ Cellular processes and signalling

$[\mathrm{A}],[\mathrm{B}],[\mathrm{J}],[\mathrm{K}] .[\mathrm{L}]$ Information storage and processing

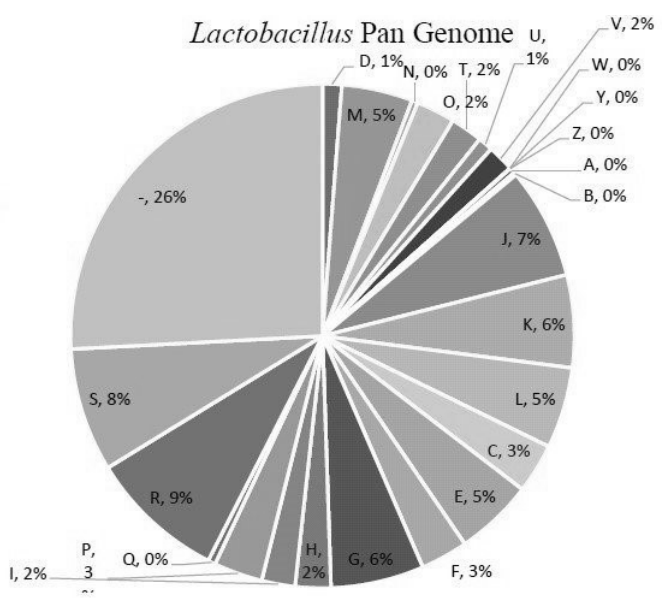

- [M] Cell wall/membrane/envelope biogenesis

- [O] Post-translational modification, protein turnover, and chaperones

- [U] Intracellular trafficking, secretion, and vesicular transport

- [W] Extracellular structures

- [Z] Cytoskeleton

- [B] Chromatin structure and dynamics

- [K] Transcription

[C] Energy production and conversion

- [F] Nucleotide transport and metabolism

- $[\mathrm{H}]$ Coenzyme transport and metabolism

- [P] Inorganic ion transport and metabolism

- [R] General function prediction only

- [-] Not assigned

$[\mathrm{C}],[\mathrm{E}],[\mathrm{F}],[\mathrm{G}],[\mathrm{H}],[\mathrm{I}],[\mathrm{P}],[\mathrm{Q}]$ Metabolism $[\mathrm{R}],[\mathrm{S}]$ Poorly characterized

Fig. 3. COG statistics of the pan genome of Bifidobacterium and Lactobacillus.

genome, $(\mathrm{G})$ is lower than in its pan genome. This indicates that carbohydrate genes differ from one another within the same genus.

\subsection{Statistical background of consensus COG ordering}

Although the difference between pan- and core-genomes depends on the definition of "the core", there is currently no straightforward definition of the core genome. No particular strain necessarily reflects the core. The core genome should be an ideal set of genes that represent the genus. Neither the 100-core nor the 80 -core reflects the true core because the strict criterion applied to the former filters out too many genes while in the latter, the threshold is too relaxed, allowing the inclusion of many auxiliary genes. Consequently, the ideal core lies in the middle. To justify our definition of the core genome, we show the trend of the size difference (the number of genes) in each COG category (Table 2). As $n$ decreases from 100, the size of the core genes increases. Each $n$-core occupies a position in the 26-dimensional space (COG functional categories, $n=26$ ), where the value on each axis is its rank, no two axes share the same value. We want to find the optimal position in this space 
Bifidobacterium Core Genome

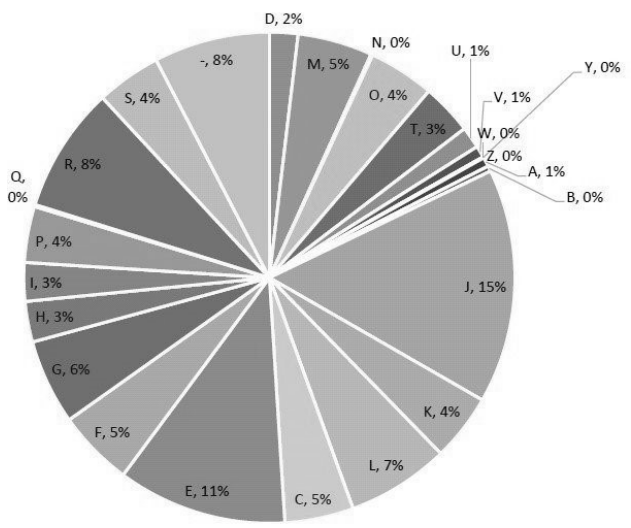

- [D] Cell cycle control, cell division, chromosome partitioning [N] Cell motility

[T] Signal transduction mechanism

- [V] Defense mechanisms

- [Y] Nuclear structure

- [A] RNA processing and modification

" [J] Translation, ribosomal structure and biogenesis

" [L] Replication, recombination and repair

" [E] Amino acid transport and metabolism

- [G] Carbohydrate transport and metabolism

- [I] Lipid transport and metabolism

- [Q] Secondary-metabolite biosynthesis, transport, and catabolism

- [S] Function unknown
Lactobacillus Core Genome

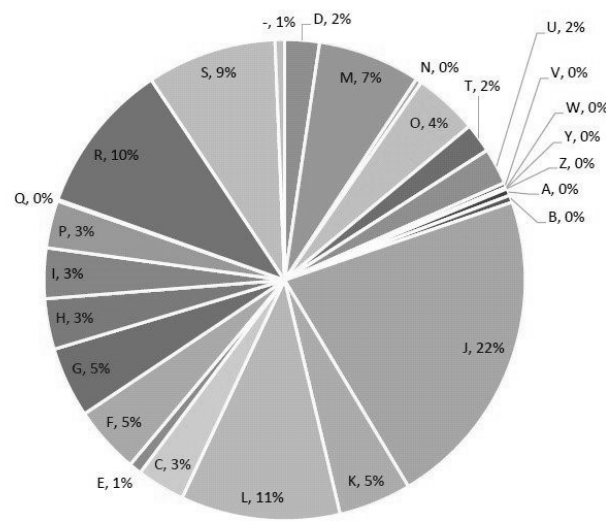

[M] Cell wall/membrane/envelope biogenesis

[O] Post-translational modification, protein turnover, and chaperones

[U] Intracellular trafficking, secretion, and vesicular transport

- [W] Extracellular structure

- [Z] Cytoskeleton

- [B] Chromatin structure and dynamics

[K] Transcription

[C] Energy production and conversion

- [F] Nucleotide transport and metabolism

- $[\mathrm{H}]$ Coenzyme transport and metabolism

- [P] Inorganic ion transport and metabolism

" [R] General function prediction only

- [-] Not assigned

$[\mathrm{D}],[\mathrm{M}],[\mathrm{N}],[\mathrm{O}],[\mathrm{T}],[\mathrm{U}],[\mathrm{V}],[\mathrm{W}],[\mathrm{Y}],[\mathrm{Z}]$ Cellular processes and signalling $[\mathrm{C}],[\mathrm{E}],[\mathrm{F}],[\mathrm{G}],[\mathrm{H}],[\mathrm{I}],[\mathrm{P}],[\mathrm{Q}]$ Metabolism

$[\mathrm{A}],[\mathrm{B}],[\mathrm{J}],[\mathrm{K}]$. [L] Information storage and processing

$[\mathrm{R}],[\mathrm{S}]$ Poorly characterized

Fig. 4. COG statistics of the core genome of Bifidobacterium and Lactobacillus.

(i.e. choosing $n$ ) based on a limited number of samplings of $n$-core $(100 \geq n \geq 83)$. Under this problem formulation, the easiest way to estimate the optimal position is by computing the average rank for each axis independently with the proviso that the averages are not necessarily integers. By choosing the $n$-core whose rank best-correlates with the average, we can avoid the problem of indeterminate ranks. The remaining problem is the extent of sampling points. From a biological perspective, 83-core was an appropriate limit for stopping our sampling because thereafter, as shown in Table 2, the number of added genes became negligible.

\subsection{Relative representation of core gene clusters in Bifidobacterium}

For the rigorous identification of the over- and under-represented COG categories in the Bifidobacterium core, we computed the OR and the $p$-value. Among 777 COG categories analyzed, 359 (46\%) were over-represented and $418(54 \%)$ were underrepresented in Bifidobacterium compared with Lactobacillus. In Bifidobacterium, 339 $(\mathrm{OR}=$ infinite $)$ COG categories were present exclusively, while $143(\mathrm{OR}=0)$ were absent exclusively. The COG database contains four major functional categories, and of these, "metabolism" was significantly over-represented in Bifidobacterium 

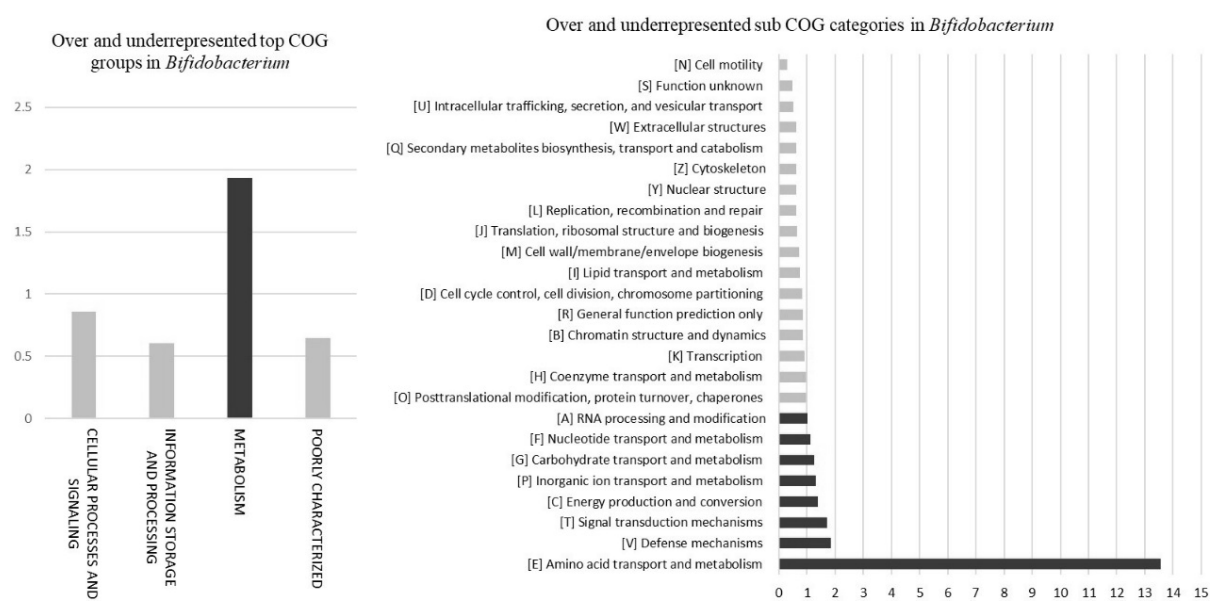

Fig. 5. Over- and under-represented COG categories: (A) top four COG groups and (B) 25 COG subcategories.

$(p<0.0001)$. "Cellular processes and signaling" and "poorly characterized" had $p<$ 0.0001 and $p<0.0053$, respectively. The under-representation of the last category "information storage and processing" was not significant (Fig. 5(a)).

The four major COG categories, "metabolism", "cellular processes and signaling", "information storage and processing" and "poorly characterized", were further divided into 25 subcategories. Of these, 17 were under-represented and 8 were over-represented (Fig. 5(b)). Among the under-represented categories, J (translation, ribosomal structure, and biogenesis; $p<0.0028$ ), L (replication, recombination, and repair; $p<0.014$ ) and S (function unknown; $p<0.0017$ ) were statistically significant (Table 3), while among the over-represented categories, only E (amino acid transport and metabolism) was significant $(p<0.0001)$. No other subcategory was statistically over- or under-represented.

Specific COG categories that were over-represented in Bifidobacterium relative to Lactobacillus mostly include COGs involved in host interaction, stress response, and complex-compounds metabolism (Table 4). In terms of gene multiplicity, glycosidases and galactosidases were multiply retained in many genomes. The table also suggests how Bifidobacterium is associated with its host and adapted to the GIT habitat. Many categories belonged to "carbohydrate transport or metabolism" (G) for competition and survival in the intestines. The presence of plasminogen-, and mucinbinding and of proteins involved in the formation of capsular or exo-polysaccharides (EPSs) is a characteristic of Bifidobacterium..$^{22}$ Other over-represented COGs included a number of protein families that facilitate the adaptation to the adverse intestinal environment such as the bile and stress resistance. An important protein detected in the core was LuxS, which is involved in biofilm formation and helps Bifidobacterium in gut colonization and protection against pathogens. ${ }^{23}$ Immunoreactive proteins like aspartokinase and surface antigens that contact host cells and 
Table 3. Significantly over- and under-represented COG categories (color coding: dark: significantly over-represented; gray: significantly under-represented).

\begin{tabular}{|c|c|c|c|c|c|c|c|}
\hline Category & $\mathrm{a}$ & $\mathrm{b}$ & $\mathrm{c}$ & $\mathrm{d}$ & OR & $p$-value & $95 \% \mathrm{CI}$ \\
\hline Cellular processes and signaling & 145 & 628 & 100 & 372 & 0.86 & 0.296 & $0.64-1.14$ \\
\hline Information storage and processing & 246 & 527 & 205 & 267 & 0.61 & $p<0.0001$ & $0.47-0.77$ \\
\hline Metabolism & 310 & 463 & 121 & 351 & 1.94 & $p<0.0001$ & $1.51-2.49$ \\
\hline Poorly characterized & 106 & 667 & 93 & 379 & 0.65 & 0.0053 & $0.47-0.87$ \\
\hline [C] Energy production and conversion & 40 & 733 & 18 & 454 & 1.38 & 0.2707 & $0.77-2.43$ \\
\hline $\begin{array}{l}\text { [D] Cell cycle control, cell division, } \\
\text { chromosome partitioning }\end{array}$ & 15 & 758 & 11 & 461 & 0.83 & 0.641 & $0.37-1.82$ \\
\hline $\begin{array}{l}\text { [E] Amino acid transport and } \\
\text { metabolism }\end{array}$ & 98 & 675 & 5 & 467 & 13.56 & $p<0.0001$ & $5.47-33.56$ \\
\hline $\begin{array}{l}{[\mathrm{F}] \text { Nucleotide transport and }} \\
\text { metabolism }\end{array}$ & 42 & 731 & 23 & 449 & 1.12 & 0.6664 & $0.66-1.89$ \\
\hline $\begin{array}{l}\text { [G] Carbohydrate transport and } \\
\text { metabolism }\end{array}$ & 47 & 726 & 23 & 449 & 1.26 & 0.3705 & $0.75-2.10$ \\
\hline $\begin{array}{l}{[\mathrm{H}] \text { Coenzyme transport and }} \\
\text { metabolism }\end{array}$ & 25 & 748 & 16 & 456 & 0.95 & 0.8813 & $0.50-1.80$ \\
\hline [I] Lipid transport and metabolism & 25 & 748 & 20 & 452 & 0.76 & 0.359 & $0.41-1.37$ \\
\hline $\begin{array}{l}{[\mathrm{J}] \text { Translation, ribosomal structure, }} \\
\text { and biogenesis }\end{array}$ & 124 & 649 & 108 & 364 & 0.66 & 0.0028 & $0.48-0.85$ \\
\hline [K] Transcription & 43 & 730 & 29 & 443 & 0.9 & 0.67 & $0.55-1.46$ \\
\hline $\begin{array}{l}\text { [L] Replication, recombination, and } \\
\text { repair }\end{array}$ & 62 & 711 & 58 & 414 & 0.62 & 0.014 & $0.42-0.90$ \\
\hline $\begin{array}{l}{[\mathrm{M}] \text { Cell wall/membrane/envelope }} \\
\text { biogenesis }\end{array}$ & 40 & 733 & 33 & 439 & 0.73 & 0.1871 & $1.45-1.16$ \\
\hline$[\mathrm{N}]$ Cell motility & 2 & 771 & 4 & 468 & 0.3 & 0.1695 & $0.05-1.66$ \\
\hline $\begin{array}{l}\text { [O] Posttranslational modification, } \\
\text { protein turnover, chaperones }\end{array}$ & 35 & 738 & 22 & 450 & 0.97 & 0.9131 & $0.56-1.67$ \\
\hline $\begin{array}{l}{[\mathrm{P}] \text { Inorganic ion transport and }} \\
\text { metabolism }\end{array}$ & 32 & 741 & 15 & 457 & 1.32 & 0.389 & $0.70-2.45$ \\
\hline $\begin{array}{l}\text { [Q] Secondary metabolites biosynthesis, } \\
\text { transport, and catabolism }\end{array}$ & 1 & 772 & 1 & 471 & 0.61 & 0.727 & $0.03-9.7$ \\
\hline [R] General function prediction only & 73 & 700 & 52 & 420 & 0.84 & 0.3706 & $0.57-1.22$ \\
\hline [S] Function unknown & 33 & 740 & 41 & 431 & 0.47 & 0.0017 & $0.29-0.75$ \\
\hline [T] Signal transduction mechanisms & 33 & 740 & 12 & 460 & 1.71 & 0.1172 & $0.87-3.34$ \\
\hline $\begin{array}{l}\text { [U] Intracellular trafficking, secretion, } \\
\text { and vesicular transport }\end{array}$ & 13 & 760 & 15 & 457 & 0.52 & 0.0893 & $0.24-1.10$ \\
\hline [V] Defense mechanisms & 6 & 767 & 2 & 470 & 1.84 & 0.457 & $0.36-9.14$ \\
\hline [A] RNA processing and modification & 10 & 763 & 5 & 467 & 1.22 & 0.7135 & $0.41-3.60$ \\
\hline [B] Chromatin structure and dynamics & 7 & 766 & 5 & 467 & 0.85 & 0.7878 & $0.26-2.70$ \\
\hline [W] Extracellular structures & 0 & 773 & 0 & 472 & 0.61 & 0.8054 & $0.01-30.83$ \\
\hline [Y] Nuclear structure & 1 & 772 & 1 & 471 & 0.61 & 0.727 & $0.03-9.77$ \\
\hline [Z] Cytoskeleton & 0 & 773 & 0 & 472 & 0.61 & 0.8054 & $0.01-30.83$ \\
\hline
\end{tabular}

modulate the immune response were also identified. There is experimental documentation of these proteins and of ABC-type sugar transporters and transaldolases in Bifidobacterium. ${ }^{24}$ Without differential analysis, representative cores would become unknown or ribosomal functions (category - S, R, or J) (Fig. 4). Unbiased selection of these functions validates the appropriateness of our core definition and the importance of comparative analysis. 
Table 4. Over-represented COG categories in Bifidobacterium (Redundancy refers to the total number with possible multiplicity in the same genome. Gene functions are based on the annotation in our reference library.).

\begin{tabular}{|c|c|c|c|c|}
\hline COG Category & OR & $\begin{array}{l}\text { Number of } \\
\text { genomes }\end{array}$ & Redundancy & Function \\
\hline $\begin{array}{l}\text { [E] COG1113 Gamma-aminobuty- } \\
\text { rate permease and related } \\
\text { permeases }\end{array}$ & infinite & 48 & 48 & $\begin{array}{c}\text { Interaction of gut microbiota } \\
\text { with the macroorganism }\end{array}$ \\
\hline [E] COG0527 Aspartokinases & infinite & 49 & 49 & Immunoreactive proteins \\
\hline $\begin{array}{l}\text { [O] COG0265 Trypsin-like serine } \\
\text { proteases }\end{array}$ & infinite & 51 & 51 & $\begin{array}{l}\text { Involved in stress response, e.g. } \\
\text { bile response }\end{array}$ \\
\hline [G] COG3345 Alpha-galactosidase & infinite & 49 & 67 & $\begin{array}{l}\text { Involved in complex carbohy- } \\
\text { drate metabolism (glycosyl } \\
\text { hydrolases (GHs)) }\end{array}$ \\
\hline $\begin{array}{l}{[\mathrm{M}] \text { COG1247 Sortase and related }} \\
\text { acyltransferases }\end{array}$ & infinite & 49 & 49 & Binding with host \\
\hline $\begin{array}{l}\text { [T] COG1854 LuxS protein } \\
\text { involved in autoinducer AI2 } \\
\text { synthesis }\end{array}$ & infinite & 51 & 51 & $\begin{array}{l}\text { Biofilm formation and host } \\
\text { colonization }\end{array}$ \\
\hline [R] COG3942 Surface antigen & infinite & 52 & 52 & Immunomodulatory activity \\
\hline [G] COG0021 Transketolase & infinite & 52 & 53 & Extracellular proteome \\
\hline [G] COG0176 Transaldolase & infinite & 52 & 52 & $\begin{array}{l}\text { Mucin-binding capability and } \\
\text { aggregation factor }\end{array}$ \\
\hline [G] COG0366 Glycosidases & infinite & 51 & 72 & $\begin{array}{l}\text { Involved in complex carbohy- } \\
\text { drate metabolism (GHs) }\end{array}$ \\
\hline $\begin{array}{l}{[\mathrm{G}] \text { COG0166 Glucose-6-phosphate }} \\
\text { isomerase }\end{array}$ & infinite & 52 & 53 & $\begin{array}{c}\text { Enzyme involve in bifidus path- } \\
\text { way (glucose metabolism) }\end{array}$ \\
\hline $\begin{array}{l}\text { [E] COG0334 Glutamate dehydro- } \\
\text { genase/leucine dehydrogenase }\end{array}$ & infinite & 49 & 49 & $\begin{array}{l}\text { Plays central roles in nitrogen } \\
\text { metabolism }\end{array}$ \\
\hline [M] COG1215 Glycosyltransferases & infinite & 52 & 52 & Involved in EPS production \\
\hline $\begin{array}{l}\text { [G] COG0033 Phosphoglucomu- } \\
\text { tase }\end{array}$ & infinite & 52 & 52 & Galactose metabolizing enzyme \\
\hline $\begin{array}{l}\text { [G] COG0580 Glycerol uptake } \\
\text { facilitator and related } \\
\text { permeases (Major Intrinsic } \\
\text { Protein Family) }\end{array}$ & infinite & 52 & 52 & $\begin{array}{l}\text { Sugar transport (non-PTS sugar } \\
\text { transport system) }\end{array}$ \\
\hline $\begin{array}{l}\text { [GM] COG1134 ABC-type poly- } \\
\text { saccharide/polyol phosphate } \\
\text { transport system }\end{array}$ & infinite & 49 & 49 & $\begin{array}{l}\text { Sugar transport (non-PTS sugar } \\
\text { transport system) }\end{array}$ \\
\hline $\begin{array}{l}\text { [GM] COG1682 ABC-type poly- } \\
\text { saccharide/polyol phosphate } \\
\text { export systems }\end{array}$ & infinite & 49 & 49 & $\begin{array}{l}\text { Sugar transport (non-PTS sugar } \\
\text { transport system) }\end{array}$ \\
\hline $\begin{array}{l}{[\mathrm{G}] \text { COG3839 ABC-type sugar }} \\
\text { transport systems }\end{array}$ & infinite & 52 & 52 & $\begin{array}{l}\text { Sugar transport (non-PTS sugar } \\
\text { transport system) }\end{array}$ \\
\hline
\end{tabular}

It can be argued whether the core genes can be computed for any set of strains to, for example, identify host-specific gene pools. Sun et al. identified cytochrome $d$ oxidases as the core of bee-specific Bifidobacterium and compared their list with other host-specific genes. ${ }^{24}$ This approach is certainly allowed but for cross-species comparisons as in this study, a statistical criterion for the core is preferable. The relationship between our strategy and bacterial host specificity remains the future problem of our study. 


\subsection{Comparison of the carbohydrate metabolism in Bifidobacterium and Lactobacillus}

An interesting application of the core in the two probiotic groups addressed the characteristics of the carbohydrate metabolism. ${ }^{25}$ We manually investigated the carbohydrate-active enzymes encoded in the two genera and confirmed the presence of GHs, glycosyl transferases (GTs), carbohydrate esterases (CEs), polysaccharide lyases (PLs) and carbohydrate-binding modules (CBMs).

According to the CAZy system classification, the pan genome of Bifidobacterium included $69 \mathrm{GHs}, 12 \mathrm{GTs}, 6 \mathrm{CEs}, 26 \mathrm{CBMs}$ but no PLs; that of Lactobacillus included $48 \mathrm{GHs}, 10 \mathrm{GTs}, 6 \mathrm{CEs}, 10 \mathrm{CBMs}$ and $15 \mathrm{PLs}$. The number of analyzed genomes in Bifidobacterium is less than a third of that in Lactobacillus, and the number of carbohydrate-active enzymes in Bifidobacterium was larger except for PLs. The most common enzymes in Bifidobacterium were four types of GHs: GH43 for xylanase and arabinanase, GH25 for muramidase, GH3 for beta-glucosidase, and GH13 for amylase. On the other hand, in Lactobacillus, the most common enzymes were GH73 for peptidoglycan hydrolase, GH25 for muramidase and GH32 for fructan hydrolase. The characteristic types of GHs reflect the different types of oligosaccharides the bacterium can metabolize: Bifidobacterium and Lactobacillus digest animal- and plantoriented sugars, respectively. While the absence of PLs in Bifidobacterium is noteworthy, there is evidence that it can metabolize polysaccharides containing uronic acids such as pectins and hemicelluloses. ${ }^{26}$ As the polysaccharides derive from bacteria, plants, and animals, it is unlikely that Bifidobacterium cannot metabolize them. Details on the sugar metabolism in Bifidobacterium are under investigation in our laboratory.

\section{Conclusions}

We created a free, curated reference library for the genus Bifidobacterium. It facilitates the accurate and consistent annotation of newly sequenced Bifidobacterium. Our comparative analysis of the probiotic genera Lactobacillus and Bifidobacterium revealed their metabolic characteristics: protein families that were over-represented in Bifidobacterium were mostly involved in the complex sugar metabolism, in host interactions, and in stress responses. These functions were in good agreement with the published data. Our analysis also detected more niche-adjusted metabolic activities such as the broad adaptability to amino acids and the polysaccharide metabolism in Bifidobacterium. Although we document the relative absence of PLs, further analysis is required to elucidate its metabolic ability with respect to polysaccharides and the host-specificity of Bifidobacterium.

\section{Acknowledgments}

Computational analysis was performed on the NIG supercomputer at the Research Organization of Information and Systems (ROIS). The research and publication 
costs for this work were supported by JSPS KAKENHI (15H05897, 17K19248, 26850054) and NIG JOINT (2017).

\section{References}

1. Lee JH, O'Sullivan DJ, Genomic insights into bifidobacteria, Microbiol Mol Biol Rev 74(3):378-416, 2010.

2. Leahy SC, Higgins DG, Fitzgerald GF, van Sinderen D, Getting better with bifidobacteria, J Appl Microbiol 98(6):1303-1315, 2005.

3. Ishibashi N, Yaeshima T, Hayasawa H, Bifidobacteria: Their significance in human intestinal health, Mal J Nutr 3:149-159, 1997.

4. Holzapfel WH, Haberer P, Geisen R, Björkroth J, Schillinger U, Taxonomy and important features of probiotic microorganisms in food and nutrition, Am J Clin Nutr 73(2 suppl):365s-373s, 2001.

5. Peres CM, Peres C, Hernández-Mendoza A, Malcata FX, Review on fermented plant materials as carriers and sources of potentially probiotic lactic acid bacteria-With an emphasis on table olives, Trends Food Sci Technol 26(1):31-42, 2012.

6. Holzapfel WH, Wood BJB (eds.), Lactic Acid Bacteria: Biodiversity and Taxonomy, John Wiley \& Sons, p. 521, 2014.

7. Di Gioia D, Aloisio I, Mazzola G, Biavati B, Bifidobacteria: Their impact on gut microbiota composition and their applications as probiotics in infants, Appl Microbiol Biotechnol 98(2):563-577, 2014.

8. O'Callaghan A, van Sinderen D, Bifidobacteria and their role as members of the human gut microbiota, Front Microbiol 7:925, 2016.

9. Bottacini F, Ventura M, van Sinderen D, O'Connell Motherway M, Diversity, ecology and intestinal function of bifidobacteria, Microb Cell Fact 13(Suppl 1):S4, 2014.

10. Ventura M, Canchaya C, Tauch A, Chandra G, Fitzgerald GF, Chater KF, van Sinderen D, Genomics of actinobacteria: Tracing the evolutionary history of an ancient phylum, Microbiol Mol Biol Rev 71(3):495-548, 2007.

11. Milani C, Lugli GA, Duranti S, Turroni F, Bottacini F, Mangifesta M, Sanchez B, Viappiani A, Mancabelli L, Taminiau B, Delcenserie V, Barrangou R, Margolles A, van Sinderen D, Ventura M, Genomic encyclopedia of type strains of the genus Bifidobacterium, Appl Environ Microbiol 80(20):6290-6302, 2014.

12. Schell MA, Karmirantzou M, Snel B, Vilanova D, Berger B, Pessi G, Zwahlen MC, Desiere F, Bork P, Delley M, Pridmore RD, Arigoni F, The genome sequence of Bifidobacterium longum reflects its adaptation to the human gastrointestinal tract, Proc Natl Acad Sci USA 99(22):14422-14427, 2002.

13. Lugli GA, Milani C, Turroni F, Duranti S, Ferrario C, Viappiani A, Mancabelli L, Mangifesta M, Taminiau B, Delcenserie V, van Sinderen D, Ventura M, Investigation of the evolutionary development of the genus Bifidobacterium by comparative genomics, Appl Environ Microbiol 80(20):6383-6394, 2014.

14. Milani C, Turroni F, Duranti S, Lugli GA, Mancabelli L, Ferrario C, van Sinderen D, Ventura M, Genomics of the genus Bifidobacterium reveals species-specific adaptation to the glycan-rich gut environment, Appl Environ Microbiol 82(4):980-991, 2016.

15. Praet J, Meeus I, Cnockaert M, Aerts M, Smagghe G, Vandamme P, Bifidobacterium commune sp. nov. isolated from the bumble bee gut, Antonie Van Leeuwenhoek 107(5):13071313, 2015.

16. Sun Z, Zhang W, Guo C, Yang X, Liu W, Wu Y, Song Y, Kwok LY, Cui Y, Menghe B, Yang R, Hu L, Zhang H, Comparative genomic analysis of 45 type strains of the genus 
Bifidobacterium: A snapshot of its genetic diversity and evolution, PLoS One 10(2): e0117912, 2015.

17. Contreras-Moreira B, Vinuesa P, GET_HOMOLOGUES, a versatile software package for scalable and robust microbial pangenome analysis, Appl Environ Microbiol 79(24):76967701, 2013.

18. Li L, Stoeckert CJ, Roos DS, OrthoMCL: Identification of ortholog groups for eukaryotic genomes, Genome Res 13(9):2178-2189, 2003.

19. Tanizawa Y, Fujisawa T, Kaminuma E, Nakamura Y, Arita M, DFAST and DAGA: Web-based integrated genome annotation tools and resources, Biosci Microbiota Food Health 35(4):173-184, 2016.

20. Park BH, Karpinets TV, Syed MH, Leuze MR, Uberbacher EC, CAZymes Analysis Toolkit (CAT): Web service for searching and analyzing carbohydrate-active enzymes in a newly sequenced organism using CAZy database, Glycobiology 20(12):1574-1584, 2010.

21. Yin Y, Mao X, Yang J, Chen X, Mao F, Xu Y, dbCAN: A web resource for automated carbohydrate-active enzyme annotation, Nucleic Acids Res 40(W1):W445-W451, 2012.

22. Salazar N, Prieto A, Leal JA, Mayo B, Bada-Gancedo JC, de los Reyes-Gavilán CG, Ruas-Madiedo P, Production of exopolysaccharides by Lactobacillus and Bifidobacterium strains of human origin and metabolic activity of the producing bacteria in milk, J Dairy Sci 92(9):4158-4168, 2009.

23. Sun Z, He X, Brancaccio VF, Yuan J, Riedel CU, Bifidobacteria exhibit LuxS-dependent autoinducer 2 activity and biofilm formation, PLoS One 9(2):e88260, 2014.

24. Górska S, Dylus E, Rudawska A, Brzozowska E, Srutkova D, Schwarzer M, Razim A, Kozakova H, Gamian A, Immunoreactive proteins of Bifidobacterium longum ssp. longum CCM 7952 and Bifidobacterium longum ssp. longum CCDM 372 identified by gnotobiotic mono-colonized mice sera, immune rabbit sera and non-immune human sera, Front Microbiol 7:1537, 2016.

25. Fushinobu S, Unique sugar metabolic pathways of bifidobacteria, Biosci Biotechnol Biochem 74(12):2374-2384, 2011.

26. Slováková L, Dusková D, Marounek M, Fermentation of pectin and glucose, and activity of pectin-degrading enzymes in the rabbit caecal bacterium Bifidobacterium pseudolongum, Lett Appl Microbiol 25(2):126-130, 2002.

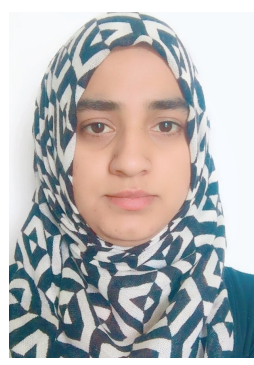

Maria Satti is currently a Graduate Student at the Department of Genetics, School of Life Science, SOKENDAI (The Graduate University for Advanced Studies), Japan. She received her Master's degree in Computational Sciences and Engineering with majors in Bioinformatics from National University of Sciences and Technology, Pakistan. Her research interests include bioinformatics and computational biology. 


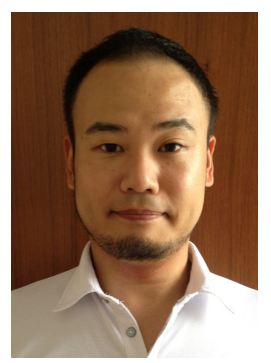

Yasuhiro Tanizawa received his Ph.D. in Bioinformatics from The University Tokyo, Japan, in 2016. After working for National Institute of Genetics (NIG) as a project researcher in "Platform for Advanced Genome Science" project, he currently works on plant and microbial genomics and its web services.

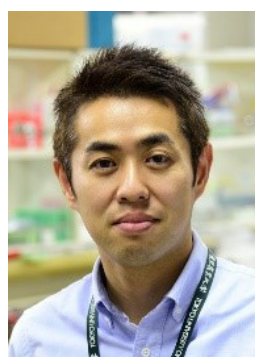

Akihito Endo received his Ph.D. in Fermentation Science from Tokyo University of Agriculture in 2005. His research interests include biochemistry and evolution of fructophilic lactic acid bacteria and impact of gut microbiota on host health.

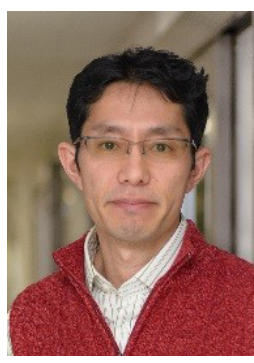

Masanori Arita is a Professor and Staff Member of DNA Data Bank of Japan, National Institute of Genetics. He also serves as the Leader of Metabolome Informatics Team at RIKEN Center for Sustainable Resource Science. He received his Ph.D. in Information Science from The University of Tokyo, and worked on bioinformatics and metabolomics in National Institute of Advanced Industrial Science and Technology (AIST), Keio University, and The University of Tokyo. 